\title{
Demonstrative experiments about gold nanoparticles and nanofilms: an introduction to nanoscience
}

\author{
Olivier Pluchery $\cdot$ Hynd Remita $\cdot$ Delphine Schaming
}

Published online: 14 November 2013

(C) The Author(s) 2013. This article is published with open access at SpringerLink.com

\begin{abstract}
An important task of the scientific community is to provide non-specialized audience with explanations about what is nanoscience. Such explanations can be given during public conferences, seminars in high schools or lab work organized with teachers. And very often, the use of an experimental illustration greatly helps to raise the interest and the curiosity of the public. The present article will describe how the authors have used five simple and visual experiments in chemistry and physics to progressively introduce different audiences into the fascination of nanoscience. One experiment is the synthesis of gold nanoparticles with the Turkevich method and shows the progressive appearance of the rubyred colour of the nanometric gold particles. The second and third experiments describe the way for modulating their colour and how to include them into a polymer and form a ruby-red coloured plastic film. The fourth experiment shows that starting from these nanoparticles, it is possible to turn them back into a yellow golden film. The last experiment is based on the optical properties of ultra-thin gold films. Using the plasmon resonance, it is possible to demonstrate that gold change colours from yellow to orange and green when a white light beam is shone on the gold interface. These visual experiments cannot be fully interpreted in front of a large audience but serve for rising curiosity.
\end{abstract}

\section{O. Pluchery}

Institut des NanoSciences de Paris, UMR 7588 CNRS, Université Pierre et Marie Curie-UPMC, 4 place Jussieu, 75252 Paris Cedex 05, France

\section{H. Remita}

Laboratoire de Chimie Physique, UMR 8000 CNRS, Université Paris-Sud, 91405 Orsay Cedex, France

D. Schaming $(\square)$

ITODYS, UMR 7086 CNRS, Sorbonne Paris Cité, Université Paris Diderot, 15 rue Jean-Antoine de Baif, 75205 Paris Cedex 13, France e-mail: delphine.schaming@univ-paris-diderot.fr
Keywords Nanoscience · Gold nanoparticles · Plasmon resonance $\cdot$ Colloids $\cdot$ Gold nanofilms $\cdot$ Teaching $\cdot$ Education

\section{Introduction}

Nanoscience awareness

Many scientists who work in the field of nanoscience have experienced that there is a gap between their daily practice and the comprehension that the common citizen can have of their research. Scientists have been trained to drive their reflections at the nanoscale, and the cutting-edge microscopes they routinely use have made them familiar with the visualization of nano-objects. Transmission electron microscopes were invented as early as 1931, and they are now commonly used to visualize the atomic structure of nanoparticles. More recently, since 1984, the scanning tunnel microscope (STM) makes possible to track individual atoms on a conductive surface and the atomic force microscope (AFM) is able to detect self-organized molecules on even non-conductive substrates. These are just examples of the way a scientist has a kind of privileged access to the "nanoworld". All these images are truly fascinating, and researchers are probably the first to be mesmerized by the beauty of the world they discover through their various microscopes. Many of these images have been used to draw the attention of the public, of the politics towards our research. Our society is puzzled to discover the amazing nanoworld that is surrounding us, or even within us. As a consequence, the nanoworld has made its way into the public subconscient.

However, once these images have made a first impression in the people's mind, they need to be explained and commented. Uncontrolled release of images or scientific information can lead to the birth of fears and mistrust towards our scientific work [1]. It is the responsibility of us, scientists, 
to make sure that the images we release convey a true message and limit the possible distortion. The situation is all the more important that the present status of nanoscience is still being questioned, by the population in most of the European countries at least. Nanoscience is still benefiting a positive image, but this is not sure it will be always so.

\section{Our responsibility for explaining nanoscience}

A recent poll issued by a well-known polling agency in France shows that in 2006, only $44 \%$ of the French population has heard about nanoscience or nanotechnology [2]. In 2010, they were $59 \%$ which is a sharp increase within only 4 years. But when one looks closer at the numbers, it turns out that among this proportion of "informed people", $22 \%$ declare that they know precisely what nanoscience is, whereas $37 \%$ have only a very rough idea. These $37 \%$ should be viewed as "goodwilling" citizens who are "ill-informed people" but eager to learn more about science. As scientists, we have a moral obligation to provide them with key concepts and explanation, so that they will turn into the group of "nanoscience-educated people". An efficient way to do so is probably that some scientists from our communities address the public through general conferences, vulgarization action in high schools, or scientific movies. Probably the most receptive public could be found in general-audience conferences and in high schools. The present article offers some support for colleagues who are ready to dedicate part of their time to this task by giving conferences and lectures. Since the impact of a scientific conference is largely magnified with the help of one or two live experiments, this paper will describe five experiments that can be presented in almost any conference hall without special equipment.

How to use the content of this article?

The aim of the present article is not to draw a typical conference guideline but rather to provide lecturers with experiments that illustrate selected features of nanoscience. The content of this paper will be most useful for teachers who want to introduce nanoscience experiments in their courses or lab teaching. It will also be useful for scientists who are invited to give general-audience conferences. Most of these experiments can be carried out in almost any conference hall, although it is useful to use a webcam and a video projector to magnify the observations. Of course, the most important in a conference is to set up a scenario where the experimental part will be just one aspect of the demonstration. Experimental evidences bring strong arguments in a demonstration. Usually, it is sufficient to present one or two experiments during a talk to definitively capture the public's attention. To maximize the impact of a conference, it is useful to focus on a few concepts of nanoscience and demonstrate their relevance. This is why, in this article, each experiment is introduced with a few sentences that can give hints towards key concept conveyed by the experiment itself. Finally, these experiments have been tested several times in real conferences [3], and the details needed to reproduce them are given in the "Experimental" part. No toxic products are used; therefore, no particular security cautions have to be taken. The cost of the experiments is low, especially for the first four ones.

\section{Experimental}

All glassware and magnetic stirrings used for gold nanoparticle (Au NP) syntheses must be cleaned with freshly prepared aqua regia solution (three parts of conc. $\mathrm{HCl}$ and one part of conc. $\mathrm{HNO}_{3}$ ). The aqua regia can be stored in a glass bottle and reused as long as the orange colour remains. The washing with aqua regia must be handled with care, wearing gloves as well as safety glasses and protective clothing. After that, items must be washed in tap water and then rinsed with distilled water (18.2 $\mathrm{M} \Omega \mathrm{cm}$ resistivity) and dried in air or in an oven.

The same ultrapure distilled water must be used for the preparation of the aqueous solutions. All chemical reagents are purchased from Sigma Aldrich. $\mathrm{KAuCl}_{4}$ can be replaced by $\mathrm{HAuCl}_{4}$. Heptane and ethanol are analytical or reagent grade and are used without purification.

\section{Experiment 1. Synthesis of gold nanoparticles}

An aqueous solution of $\mathrm{KAuCl}_{4}$ is heated to the boiling point in an Erlenmeyer, and then, a sodium citrate aqueous solution is added with vigorous magnetic stirring. This solution is heated during about $10 \mathrm{~min}$, and the obtained red solution is finally left cooling. In Table 1, the experimental conditions to obtain Au NPs with two well-defined sizes are given.

Experiment 2. Change of colour by aggregation with salt

At least $0.5 \mathrm{~g}$ of $\mathrm{NaCl}$ in $10 \mathrm{~mL}$ of distilled water is dissolved, or a saturated solution $(>360 \mathrm{~g} / \mathrm{L}$, i.e. $6.1 \mathrm{~mol} / \mathrm{L})$ is used. A small amount (about 3-4 mL) of the Au NPs solution is put in two test tubes. One tube is used as a colour reference. To the other tube, 5-10 drops of the saturated $\mathrm{NaCl}$ solution are added. The colour of the solution changes in the second tube. This experiment could be done in a cuvet with the UV-visible spectrum recorded after each addition.

Experiment 3. Polymeric film containing gold nanoparticles

A concentrated solution of poly(vinyl alcohol) (PVA) (1 M in monomer) is prepared by dissolving $440 \mathrm{mg}$ in $10 \mathrm{~mL}$ of distilled water. The beaker containing PVA in water is put in a water bath, and the solution is heated to boiling under 
Table 1 Experimental conditions to obtain Au NPs with 15 and $30 \mathrm{~nm}$ of diameter

\begin{tabular}{llll}
\hline NP diameter $(\mathrm{nm})$ & Gold salt solution & Sodium citrate solution & Ratio of citrate to gold \\
\hline 15 & $20 \mathrm{~mL}$ at $0.25 \mathrm{mM}(1.9 \mathrm{mg})$ & $1 \mathrm{~mL}$ at $34 \mathrm{mM}(1 \mathrm{wt} \%)$ & 6.8 \\
30 & $20 \mathrm{~mL}$ at $1 \mathrm{mM}(7.6 \mathrm{mg})$ & $0.8 \mathrm{~mL}$ at $34 \mathrm{mM}(1 \mathrm{wt} \%)$ & 1.4 \\
\hline
\end{tabular}

stirring. The water bath prevents the PVA from sticking on the bottom of the beaker. A homogeneous solution of PVA is obtained after a few tens of minutes. Then, $2 \mathrm{~mL}$ of the colloidal $\mathrm{Au}$ NPs solution is added to $3 \mathrm{~mL}$ of the concentrated PVA solution. After stirring, the mixture is put in a crystallizing dish (of about 3-4 cm in diameter) and heated at 70$80{ }^{\circ} \mathrm{C}$ in order to evaporate water. Care should be taken to avoid overheating, since this can lead to non-homogeneous distribution of the Au NPs in the film. A thin film of PVA containing Au NPs is obtained after a few hours of drying.

Au NPs can also be induced directly in PVA solution containing $\mathrm{KAuCl}_{4}$ [4]: PVA acts as reducing and stabilizing agent [5]. The Au NPs are prepared as follows: in a typical experiment, $4 \mathrm{~mL}$ of $2.4 \mathrm{mM} \mathrm{KAuCl}{ }_{4}$ aqueous solution is added into $80 \mathrm{~mL}$ of $0.24 \mathrm{M}$ (repeating unit) PVA aqueous solution. Then, the mixture is heated at $80{ }^{\circ} \mathrm{C}$ for several hours, yielding a purple solution. The film is then prepared in a crystallizing dish as described before.

Experiment 4. Formation of a metallic gold film from colloidal gold nanoparticles

Forty millilitres of an aqueous Au NP solution is centrifugated (around 3,000 rpm, until the supernatant becomes pale pink). The supernatant is then removed, and the concentrated aliquot of Au NPs is redissolved in $0.8 \mathrm{~mL}$ of ethanol. A heptane/ water biphasic system is prepared in small glassware with ca. 3-4 $\mathrm{cm}^{2}$ of section. In agreement with the densities of these two solvents, the water is located below and the heptane above. The concentrated ethanol solution of Au NPs is then added by small injections in the organic phase near the organic/aqueous interface until a golden film appears at the interface.

Experiment 5. Surface plasmon resonance and the colour of gold

The surface plasmon wave is an electromagnetic wave that arises at the interface between a metal and an insulating medium (dielectric) and whose structure is that of an evanescent wave. It can be seen as a wave confined very close to the metallic surface [6]. In the case of the gold/air interface and at a wavelength of $633 \mathrm{~nm}$, this wave is confined within a 30nm-thick layer close to the interface and crawls along the gold surface over a distance of $10 \mu \mathrm{m}$. Its excitation is possible only with geometries capable of generating large wave vectors parallel to the interface. The most common geometry is the Kretschmann geometry shown in Fig. 1 where the impinging wave is generated by total internal reflection on a glass prism as depicted in Fig. 1b. The gold film should be deposited on this face of the prism. The experiment consists in measuring the reflected intensity as a function of incidence angle when the light is $p$-polarized. If one measures this intensity when a helium-neon laser in used $(\lambda=633 \mathrm{~nm})$, the reflected intensity exhibits a sharp minimum for an angle of ca. $30^{\circ}$ as plotted in the graph of Fig. 1c [7]. From this plot, it is easy to understand that if the angle is set at $30^{\circ}$, and if a white light beam from a halogen lamp is used instead of a monochromatic laser beam, the red component of light will not be reflected. Therefore, the observed resulting beam will appear green. And by adjusting the angle, it is possible to turn off various colours, and the colour of the observed beam will also vary. This is a typical effect arising from the confinement of an optical wave into a nanometer-thin gold film. A setup that exhibits this effect is described in details in a publication [7] and is available from a small company as a lab work for master students [8].

\section{Experiment 1. Synthesis of gold nanoparticles}

While gold is known since a long time for its golden colour so appealing in jewellery, gold is also used since antiquity as red dye. For instance, Au NPs have been found in several red glasses or on porcelains decorated with red or pink enamels dating from ancient times, for instance, in Roman glassworks (such as the famous Lycurgus Cup dating from the fourth century CE) or in the Chinese "Famille Rose" porcelain dating from the eighteenth century [9-11]. Indeed, bulk gold reflects the yellow colour and appears golden-coloured, whereas at the nanoscale gold appears generally red. Michael Faraday, fascinated by this ruby colour of colloidal gold solutions, was the first scientist who has discovered that the optical properties of gold colloids differ from those of the corresponding bulk metal. He reported on the first synthesis of Au NPs in solution in 1857 [12]. These colloids were obtained by reduction of gold salts by white phosphorous [13]. Later, the specific colours of metal colloids were explained by Mie [14].

This phenomenon is due to the well-known plasmon resonance also called local surface plasmon resonance (LSPR), which can be explained by a confinement of the electromagnetic wave associated with the light inside the NPs. Indeed, when its wavelength is greater than the size of the NPs, the 
Fig. 1 a Image of a setup used for generating surface plasmon waves. b The central part of the setup is a glass prism where a 50 $\mathrm{nm}$-thick gold film was deposited. When a $p$-polarized laser beam is shone on this gold film, the reflected intensity exhibits a sharp minimum for one given angle of incidence. c The corresponding plot of the reflected intensity as a function of the incidence angle is shown. This minimum indicates that the energy is transferred to the surface plasmon wave

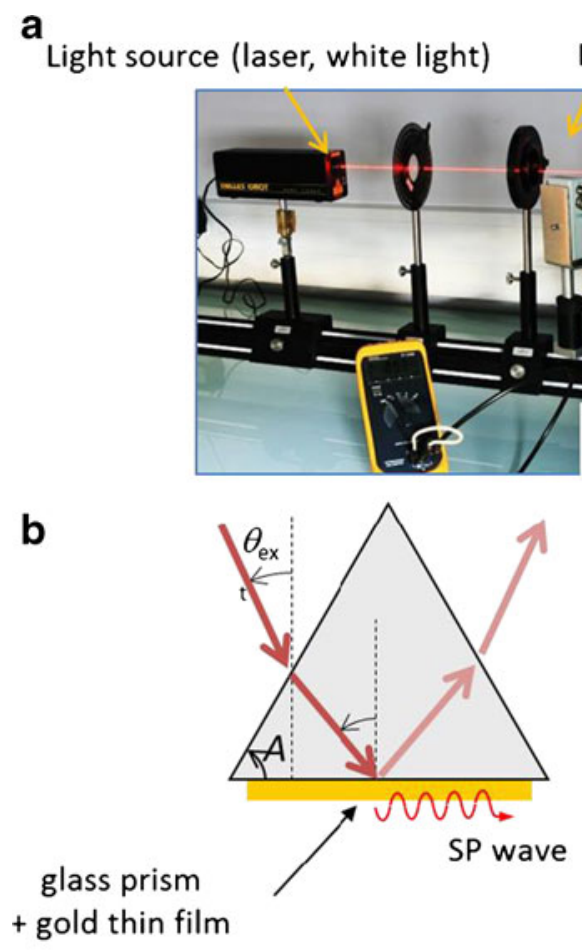

polarizer

Prism + gold thin film mounted on a goniometer stage
Photodiode connected to a multimeter

\section{C}

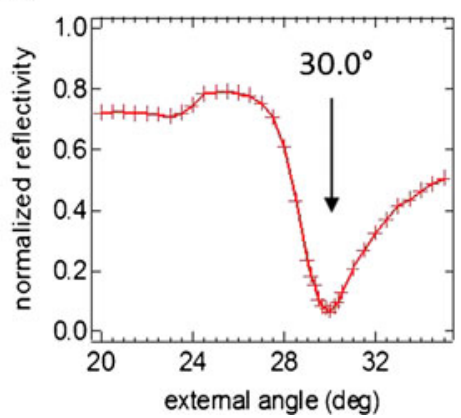

whole NP feels a uniform and oscillating electric field, and consequently electrons oscillate in phase. Nevertheless, this collective oscillation of the electrons is constrained by the reduced dimensions of the NP in which they are confined, leading to a significant absorption of the wavelengths around green. Then, NPs appear with the complementary colour, which is red.

This experiment introduces the audience into the idea that at the nanoscale, basic properties, such as colour, turn out to be very different from what happens in the "macro-world". Therefore, nanoscience focuses on properties linked not only to a material but to its specific size. And sometimes, the changes of properties at the nanoscale have consequences visible at our human scale. In the present case, the assembly of gold atoms into NPs of ca. 1,000 atoms changes the colour of gold. This message is always puzzling for non-scientific audience.

Nowadays, among the different ways of synthesis of spherical Au NPs, Turkevich's method is the most known and the easiest to perform [15]. This method is based on the reduction of an $\mathrm{Au}$ (III) salt by sodium citrate, as described in the experimental part (experiment 1). The reaction starts by boiling a gold salt aqueous solution (pale yellow). A few minutes after the addition of sodium citrate, the mixture becomes firstly uncoloured, then grey, violet, and finally burgundy red (Fig. 2a). In this reaction, the sodium citrate is used not only as reducing agent, but also as stabilizing agent. Indeed, its stabilizing role restrains the growth of the NPs and controls the NP diameter. Thus, the size of the NPs can be modulated by changing the concentration of the gold salt solution and the ratio of the quantity of gold salt to the quantity of sodium citrate $[16,17]$. The sizes of the NPs described in this experiment are 15 and $30 \mathrm{~nm}$ and has been verified by transmission electronic microscopy (TEM) (Fig. 2b)

Diffusion (or scattering) of light by the Au NPs can be evidenced with a laser beam pointed to the solution (Fig. 2c) and can be compared with a similar experiment using an aqueous solution containing an organic red dye (rhodamine or Congo red for instance) for which diffusion is not detectable (Fig. 2d). When light is scattered by single molecules such as dye molecule, the efficiency of the phenomenon is very low (usually quantified with the scattering cross section) and is barely visible by eyes (Fig. 2d). However, this cross section grows as the volume of the object, and with a particle of $30 \mathrm{~nm}$ whose diameter is 100 times larger than a molecule (volume $10^{6}$ larger), the scattering process becomes easily visible as demonstrated in Fig. 2c. Moreover, Fig. 2c shows that if the laser wavelength is tuned to the plasmon resonance, the scattering is even stronger. The green laser beam is quickly damped when going through the NP suspension, whereas the red laser light is scattered less efficiently, but over a longer path.

Many other chemical methods of synthesis exist [18] and can lead to different shapes of NPs: nanorods [19, 20], nanopyramids [21], nanocubes, etc. Physical chemical syntheses such as photolytic and radiolytic methods can also be used, and generally lead to a better control of the size of the NPs [18, 22, 23]. Chemical and optical properties of Au NPs depend on their size, their shape, their aggregation state and their local environment. Such Au NPs have applications for example in catalysis (oxidation of $\mathrm{CO}$ ), medicine 

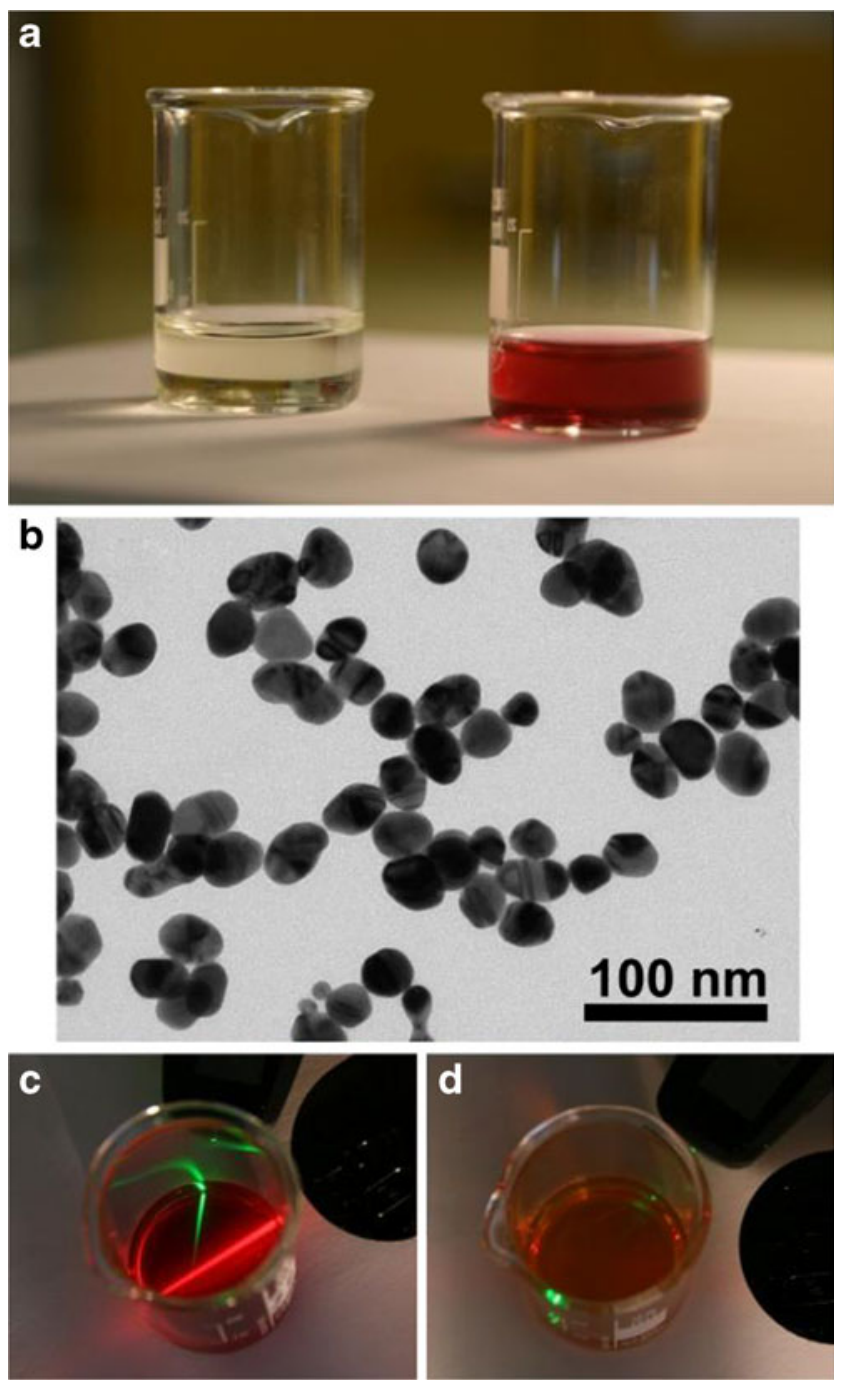

Fig. 2 a Pale yellow $\mathrm{Au}(\mathrm{III})$ salt $(1 \mathrm{mM})$ (left) and red Au NP (right) solutions. b TEM image of Au NPs with $30 \mathrm{~nm}$ of diameter used in solution B. c Green and red laser beams diffused in an Au NP solution. d Comparison with a similar experiment with a red dye solution

(photothermal therapy and radiotherapy), plasmonics and electronics $[9,24,25]$.

\section{Experiment 2. Colour change of nanoparticles by aggregation with a salt}

Upon addition of $\mathrm{NaCl}$, the initially red colour of the $\mathrm{Au} \mathrm{NP}$ solution turn to blue grey (experiment 2).

This experiment illustrates the extreme sensitivity of the coloration property with phenomena occurring at the nanoscale: if NPs are driven close to each other, the plasmon resonance is altered, and the colour changes (Fig. 3a, b).

The surface of Au NPs is charged. This induces repulsive forces between the NPs preventing them from aggregation: the energy barrier is too strong for interaction to occur between particles. However, upon addition of $\mathrm{NaCl}$, this energy barrier is reduced allowing the Au NPs to interact and aggregate. Dissolved salts, or electrolytes in general, are able to screen the repulsive electrostatic forces caused by the citrate layer: indeed, the positive charges of the electrolyte associate with the negative charges on the surfaces of the NPs. CryoTEM experiments, which consist in freezing the NPs after deposition onto an analysis grid by dipping it in a cooling media (liquid nitrogen), allowing to clearly show initially well-dispersed $\mathrm{Au}$ NPs in solution (before addition of $\mathrm{NaCl}$ ) and their aggregation after addition of $\mathrm{NaCl}$, but the nanoparticles keep their shape (Fig. 3c, d). This aggregation causes surface plasmon coupling which induces a shift in the surface plasmon resonance to a higher wavelength, resulting in a change in colour of the solution from red to blue in our case (Fig. 3a). These experiments can be done in an optical cell, and with a spectrophotometer, and students can monitor the NP aggregation by the UV-visible absorption of the solution (Fig. 3b). The students can then associate the absorbed wavelength with the observed colour of the solution.

These experiments show that individual small Au NPs appear red; however, when the particles aggregate together, the plasmon resonances can couple, and the colour changes to blue.

This property is used to develop biosensors. Au NPs can be easily functionalized. Thus, binding of Au NPs to biomolecules offers a promising approach for facile tracking of desired targets in aqueous samples. Antibody/antigen pairs can selectively link to the NPs. By modifying the surfaces of the NPs to incorporate these biomolecules, binding events can be detected by a change in solution colour. The most familiar example of NPs in sensing is the home pregnancy test based on detection of the hormone pregnancy ( $\beta$-HCG hormone) [26]. Tests for DNA detection take also advantage of surface plasmon resonance changes [27]. For example, one kind of DNA test looks for certain bases. In this test, NPs are present as large aggregates displaying a blue colour. If the complementary DNA base is present, the NPs will preferentially bind to that base instead of each other, and the aggregates will dissolve inducing a deep red colour. We can detect the colour changes associated with DNA binding in about 2 min, making for a rapid assay. Au NPs functionalized with antibodies can be also used for rapid detection of virus (such as $\mathrm{A} / \mathrm{H} 5 \mathrm{~N} 1$ ) [28]. Another example is the use of Au NPs for sensitive diagnostic tests and novel treatments in the detection of Alzheimer's disease by finding a protein in spinal fluid [29].

\section{Experiment 3. Polymeric film containing gold nanoparticles}

A thin film of Au NPs dispersed in PVA can be obtained by simply drying the gold colloid in the presence of dissolved PVA (at high concentration) (experiment 3). Au NPs will be randomly distributed within the PVA film (TEM images not 
Fig. 3 a Au NP solution without $\mathrm{NaCl}$ (left) and with $\mathrm{NaCl}$ (right). b UV-visible absorption spectra of aqueous solutions of Au NPs with $15 \mathrm{~nm}$ of diameter, without and with $\mathrm{NaCl}$ (optical path= $2 \mathrm{~mm}$ ). Cryo-TEM images of $\mathbf{c}$ non-aggregated Au NPs (without $\mathrm{NaCl}$ ) and of $\mathbf{d}$ aggregated $\mathrm{Au}$ NPs (with $\mathrm{NaCl}$ )

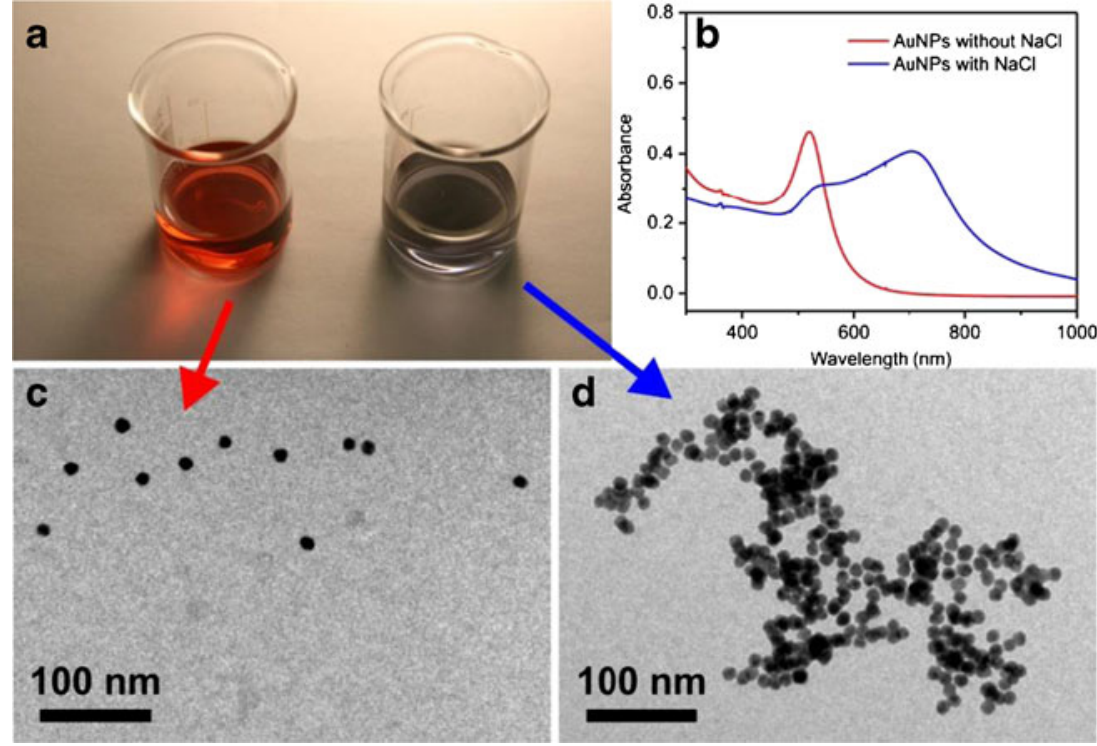

shown here). The film appears purple, because the index of refraction of the medium surrounding the Au NPs is changed: from $n=1.33$ (in aqueous solution) to $n \sim 1.7$ (in the PVA film) (Fig. 4).

This experiment demonstrates that Au NPs keep their unusual optical properties, even outside of the preparation solution. It makes possible to use their nanoscale properties for various applications in the daily life. Indeed, composite materials made of NPs and polymeric matrices are suitable candidates for biomedical applications, magnetic storage, optoelectronic and electronic devices.

\section{Experiment 4. Formation of a metallic gold film from colloidal gold nanoparticles}

While Au NPs are generally red, can we obtain again a golden appearance if we build bulk gold by aggregating and coalescing a lot of NPs? The experiment proposed in this part consists in the formation of a golden metallic film at the interface

Fig. 4 PVA film containing $\mathrm{Au}$ NPs of $30 \mathrm{~nm}$ of diameter

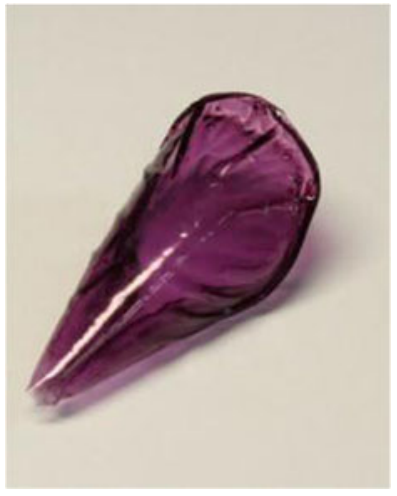

Fig. 5 Images of metallic gold films formed at the interface between the two non-miscible liquids

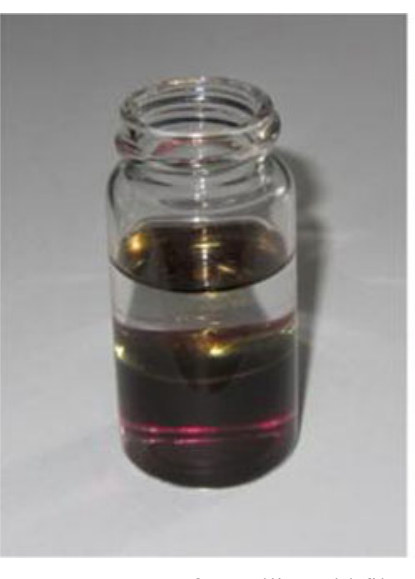

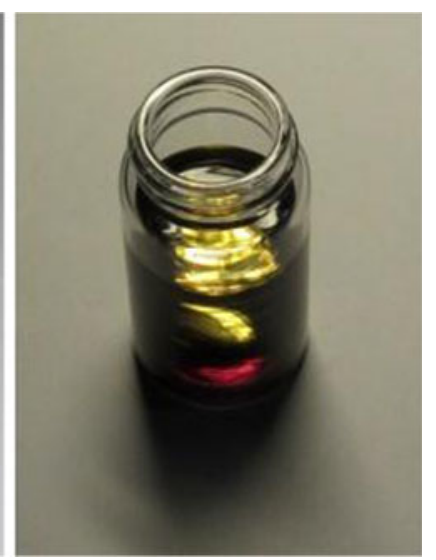

between two immiscible liquids (Fig. 5), using a colloidal Au NP solution (experiment 4).

This experiment consists in using a water/heptane biphasic system and adding an ethanol solution of colloidal Au NPs at the interface between the two immiscible liquids [30]. Indeed, when Au NPs are redissolved in ethanol, the surface charge density of the NPs decreases, most likely due to the competitive adsorption of alcohol with citrate on the NPs. The solubility of the $\mathrm{Au}$ NPs in the aqueous phase is decreased when they diffuse towards the water/heptane interface. Moreover, when a water-miscible solvent with a lower dielectric constant, such as ethanol, is added at the interface, the interfacial energy between the two phases decreases even more, leading to the formation of the mirror-like gold film at the interface [31]. 


\section{Experiment 5. Surface plasmon resonance and the colour of gold}

The usual colour of bulk gold is yellow, which itself is a nonusual property for metals since they are all grey except gold and copper. This yellow colour is due to the so-called interband transitions of gold that occur in the blue region of the visible spectrum [32]. As a consequence, the reflected light is deprived of its blue component and appears yellow. However, when the thickness of a gold layer decreases down to the nanometer scale, light behaves differently. This phenomenon can be shown in a demonstrative way in the case of the surface plasmon resonance using the setup described in Figs. 1a and 5a. The gold thin film which is attached on the prism should be illuminated with a beam light from a white light lamp. The excitation beam enters the prism through one face, and the reflected beams exits through another face and is observed on a screen (Fig. 6a, b). The experiment consists in slowly rotating the prism: the beam reflected from the thin film goes through a range of different colours that can be easily viewed on a white screen and that are absolutely unusual for gold. This change of colour of a reflected beam is the visible manifestation of the presence of the surface plasmon wave [6]. The energy at one wavelength of the impinging wave is transferred to the surface plasmon wave, and the reflected beam is strongly attenuated. As a consequence, this wavelength is missing in the reflected beam, and the observed colour changes (Fig. 6c). For example, if the angle of the rotation stage is set at $30^{\circ}$ in our setup, the surface plasmon wave is generated for the red wavelength, and the reflected beam will be green (complementary colour to red). It helps demonstrate that light behaves very differently when interacting with nano-objects. This experiment can also lead the audience to plasmonics and to the fabrication of some kinds of ultra-sensitive biosensors. An impressive way to demonstrate that this plasmon wave is a very sensitive sensor is to blow gently towards the gold film. The slight optical index change induced by the air flow makes the colours fluctuate. It can help in understanding that if a layer of molecules adsorbs on the gold film, the index change will also be detected by the surface plasmon wave.

\section{Conclusion}

A set of five easy experiments has been proposed as an introduction to key concepts of nanoscience targeting a public audience of non-specialists. They are all based on the use of gold either as nanoparticles or in nanometric thin films. The main message is that usual material exhibits unexpected properties when one of their dimensions is nanometric. This article explores more specifically the colour modulations of gold at the nanometric size. The first four experiments are based on gold nanospheres of 15 to $30 \mathrm{~nm}$ in diameter that are prepared according to the Turkevich method. In this case, gold adopts a magnificent ruby-red colour. Experiments 1, 2, and 3 describe the chemical preparation, the way for modulating their colour and how to include them into a polymer film. Experiment 4,
Fig. 6 Experimental setup for exciting surface plasmon wave on a thin gold film (50 $\mathrm{nm}$ in thickness) placed on one face of a glass prism. The reflected beam changes colour when the prism is rotated. a The excitation is achieved with white light that needs to be $p$-polarized and is reflected by the prism. The resulting colours are observed on the screen. $\mathbf{b}$ At a given incidence angle, the incoming light is able to excite the surface plasmon wave for one wavelength, the remaining wavelengths being reflected. $\mathbf{c}$ The photo shows the range of colours reflected by the glass prism (which is visible in the right part). This picture was taken with a long exposure time so that five different angles of the rotation stage were simultaneously captured a

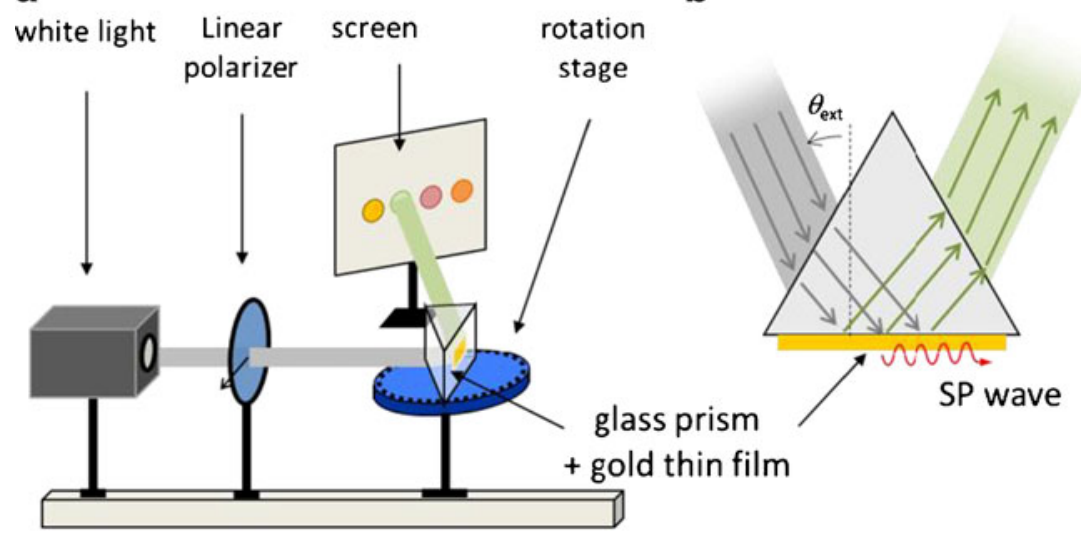

C 
based on the use of immiscible liquids, is a striking experiment that shows that starting from these nanoparticles, it is possible to turn them back into a gold film that recovers its conventional yellow colour. Finally, the last experiment plays with the optical properties of the surface plasmon wave. These experiments can be used for teaching in lab courses with undergraduate students after some adaptation. Of course when used in conferences, they are always capturing the public attention if they are conducted as live experiments, although it demands some training so that the effect comes up at the right moment. This is always a very rewarding feeling for us scientists to be able to raise a fascination for scientific work and contribute to attract young people into the scientific community.

It has to be noted that some experiments proposed in this paper have been tested many times with success in school, in partnership with the Nano-School (www.nano-ecole.fr) project. This action aims to educate young people about nanoscience and give them some basics. These experiences around gold nanoparticles are particularly suitable in this context. This partnership has also helped to develop a kit for synthesizing nano particles, for teachers of middle and high schools (www.jeulin.fr).

Acknowledgments The authors thank Patricia Beaunier (Laboratoire de Réactivité de Surface, Université Pierre et Marie Curie) for the TEM observations and Jean-Michel Guigner (Institut de Minéralogie et de Physique des Milieux Condensés, Université Pierre et Marie Curie) for the Cryo-TEM studies. Finally, this paper is in debt to Don Eigler, a prominent physicist, known for his pioneering work in STM. One of the authors attended a keynote given by Don Eigler in 2006 in Basel (Switzerland) entitled "Computation in Small Structures: a Long Road to an Uncertain Destination". The content of the conference was totally unexpected since Don Eigler did not talk about computation at all, but about the urgent need for scientists to address the issue of informing the public, the journalists, the companies and the politics. This paper shows that his call has been heard.

Open Access This article is distributed under the terms of the Creative Commons Attribution License which permits any use, distribution, and reproduction in any medium, provided the original author(s) and the source are credited.

\section{References}

1. Carenco S (2012) Développons les nanomatériaux! Rue d'Ulm, Paris

2. IPSOS (2010) Les Français et les nanotechnologies. Poll issued by IPSOS from a sample of 1013 people aged greater than 15

3. Pluchery O (2007) Filmed conference: "Propriétés optiques de l'or nanométrique: la plasmonique" http://www.dailymotion.com/ webinsp\#video=x9mkdt

4. Sun C, Qu R, Ji C, Meng Y, Wang C, Sun Y, Qi L (2009) Preparation and property of polyvinyl alcohol-based film embedded with gold nanoparticles. J Nanoparticle Res 11(4):1005-1010

5. Gachard E, Remita H, Khatouri J, Keita B, Nadjo L, Belloni J (1998) Radiation-induced and chemical formation of gold clusters. New $\mathrm{J}$ Chem 22:1257-1265
6. Novotny L, Hecht B (2006) Principles of nano-optics. Cambridge University Press, Cambridge

7. Pluchery O, Vayron R, Van K-M (2011) Laboratory experiments for exploring the surface plasmon resonance. Eur J Phys 32:585

8. Pluchery O, Ney P (2012) Experimental setup for studying surface plasmon resonance in lab courses. This Lab work equipment is sold by the company DIDA Concept, France. http://www.didaconcept. com. Accessed 6 Nov 2013

9. Schaming D, Remita H (2013) Nanotechnology: from the ancient time to nowadays. Found Chem (in press)

10. Louis C (2012) Gold nanoparticles in the past: before the nanotechnology era. In: Louis C, Pluchery O (eds) Gold nanoparticles for physics, chemistry and biology. World Scientific Publishing, London

11. Freestone I, Meeks N, Sax M, Higgitt C (2007) The Lycurgus Cupa Roman nanotechnology. Gold Bull 40(4):270-277

12. Faraday M (1857) Experimental relations of gold (and other metals) to gold. Phil Trans R Soc London 147:145-181

13. Thompson D (2007) Michael Faraday's recognition of ruby gold: the birth of modern nanotechnology. Gold Bull 40(4):267-269

14. Mie G (1908) Beiträge zur Optik trüber Medien, speziell kolloidaler Metallösungen. Ann Phys 25:377-445

15. Turkevich J, Stevenson PC, Hillier J (1951) A study of the nucleation and growth processes in the synthesis of colloidal gold. Discuss Faraday Soc 11:55-75

16. Zabetakis K, Ghann WE, Kumar S, Daniel M-C (2012) Effect of high gold salt concentrations on the size and polydispersity of gold nanoparticles prepared by an extended Turkevich-Frens method. Gold Bull 45:203-211

17. Ji X, Song X, Li J, Bai Y, Yang W, Peng X (2007) Size control of gold nanocrystals in citrate reduction: the third role of citrate. J Am Chem Soc 129(45):13939-13948

18. Zhao P, Li N, Astruc D (2013) State of the art in gold nanoparticle synthesis. Coord Chem Rev 257:638-665

19. Jana N, Gearheart L, Murphy C (2001) Seed-mediated growth approach for shape-controlled synthesis of spheroidal and rod-like gold nanoparticles using a surfactant template. Adv Mater 13:1389-1393

20. Nikoobakht B, El-Sayed M (2003) Preparation and growth mechanism of gold nanorods (NRs) using seed-mediated growth method. Chem Mater 15:1957-1962

21. Liu M, Guyot-Sionnest P (2005) Mechanism of silver(I)-assisted growth of gold nanorods and bipyramids. J Phys Chem B 109: 22192-22200

22. Abidi W, Remita H (2010) Gold based nanoparticles generated by radiolytic and photolytic methods. Recent Pat Eng 4(3):170-188

23. Torigoe K, Esumi K (1992) Preparation of colloidal gold by photoreduction of tetracyanoaurate(-)-cationic surfactant complexes. Langmuir 8:59-63

24. Hainfeld JF, Slatkin DN, Smilowitz HM (2004) The use of gold nanoparticles to enhance radiotherapy in mice. Phys Med Biol 49(18):N309-315

25. Sardar R, Fuston AM, Mulvaney P, Murray RW (2009) Gold nanoparticles: past, present, and future. Langmuir 25(24): $13840-13851$

26. Tanaka R, Yuhi T, Nagatani N, Endo T, Kerman K, Takamura Y, Tamiya E (2006) A novel enhancement assay for immunochromatographic test strips using gold nanoparticles. Anal Bioanal Chem 385(8):1414-1420

27. Liu J, Lu Y (2006) Preparation of aptamer-linked gold nanoparticle purple aggregates for colorimetric sensing of analytes. Nat Protoc 1(1):246-252

28. Pham VD, Hoang H, Phan TH, Conrad U, Chu HH (2012) Production of antibody labeled gold nanoparticles for influenza virus H5N1 diagnosis kit development. Adv Nat Sci: Nanosci Nanotechnol 3(4):045017

29. Georganopoulou DG, Chang L, Nam J-M, Thaxton CS, Mufson EJ, Klein WL, Mirkin CA (2005) Nanoparticle-based detection in 
cerebral spinal fluid of a soluble pathogenic biomarker for Alzheimer's disease. Proc Natl Acad Sci 102(7):2273-2276

30. Fang P-P, Chen S, Deng H, Scanlon MD, Gumy F, Lee HJ, Momotenko D, Amstutz V, Cortes-Salazar F, Pereira CM, Yang Z, Girault HH (2013) Conductive gold nanoparticle mirrors at liquid/ liquid interfaces. ACS Nano 7(10):9241-9248
31. Schaming D, Hojeij M, Younan N, Nagatani H, Lee HJ, Girault HH (2011) Photocurrents at polarized liquid/liquid interfaces enhanced by a gold nanoparticle film. Phys Chem Chem Phys 13:17704-17771

32. Pyykkö P (2004) Theoretical chemistry of gold. Angew Chem Int Ed 43(34):4412-4456 